\title{
THE HIPPARCOS MISSION AND THE RE-REDUCTION OF BELGRADE ZENITH-TELESCOPE OBSERVATIONS
}

\author{
G. DAMLJANOVIĆ \\ Astronomical Observatory \\ Volgina 7, 11050 Belgrade, Yugoslavia \\ E-mail: gdamljanovic@aob.aob.bg.ac.yu
}

\begin{abstract}
We prepared the results (in a computer-readable form) of the Belgrade ZT observations made in the period 1949-1985 and finished their new reduction in the FK5 reference frame.
\end{abstract}

\section{Introduction}

At the XXI IAU General Assembly, Buenos Aires 1991, Commission 19 of the IAU, "Rotation of the Earth", formed the Working Group on Earth Rotation in the HIPPARCOS reference frame-WG ERHRF to collect the past observations and to analyse them in that reference system. Our investigations are in accordance with it. The WG ERHRF has set up a list of the best observations performed in the past. The Belgrade Observatory is on that list (Vondrák, Feissel and Essaifi, 1992) with the observations in the period 1949-1990 obtained with ZT (the visual zenith-telescope AskaniaBamberg No $77241,110 / 1287 \mathrm{~mm}$ ) by applying Talcott's method.

The Old Belgrade Latitude Programme - OP (Djurković,Ševarlić, Brkić, 1951) was observed in the period 1949-1960. The New Belgrade Latitude Programme - NP (Ševarlić and Teleki, 1960) was started in 1960 and the observations are still carried out. We used the PPM Star Catalogue (Röser \& Bastian, 1991) for the re-reduction.

\section{Procedure and results}

The re-reduction is in accordance with MERIT standards (Melbourne et al., 1983). The new IAU(1976) coordinate system of astronomical constants, 
the IAU(1980) nutation model, the new dynamical reference system (JPL DE200/LE200 Ephemeris, 1984), and the FORTRAN programme for refraction (Abalakin, 1985) are used. The trigonometric stellar parallaxes (Jenkins, 1952) and the stellar radial velocities (Wilson, 1953) are used for the calculation of the apparent places of OP and NP stars.

We used the Student-Fisher criterion for eliminating the excessive instantaneous latitudes resulting from some Talcott's pairs.

The polar motion was eliminated from the material and the observations were brought in accordance with the mean pole BIH1979. After that we made determinations of the systematic errors of declinations and proper motions of Talcott's pairs and (sub)groups of OP and NP.

We used the new instrument's constants applied in the re-reduction (the angular value of the micrometer screw revolution, the angular division values and the temperature coefficients of the Talcott's levels) and the numerous systematic errors are taken into account, see (Damljanović, 1994, 1995).

\section{Conclusion}

The mean error of the instantaneous latitude from one Talcott's pair is less than before. From the preliminary ZT observations made 1947 the mean error was $\pm 0 . " 255$ (Djurković, Ševarlić, Brkić, 1951). The mean error of the OP (1949-1960) was $\pm 0 . " 220$ (Ševarlić and Teleki, 1960), and of the NP was $\pm 0 . " 272$ (1960-1965.5) and $\pm 0 . " 146$ (1969-1974) (Grujić et al. 1989). After our re-reduction the mean error of the OP is $\pm 0 . " 199$ (1949-1960) and the mean error of the NP is $\pm 0 . " 148(1960-1985)$.

\section{References}

Abalakin, V. K. (1985), Refraction Tables of Pulkovo Observatory (V edition), NAUKA, Leningrad, GAO AN SSSR.

Djurković, P., Ševarlić, B., Brkić, Z. (1951) Publ. Obs. Astron. Belgrade, 4.

Damljanović, G.(1994) Bull. Astron. Belgrade, 150, pp. 29.

Damljanović, G.(1995) Bull. Astron. Belgrade, 152, pp. 71.

Grujić, R., Djokić, M., Jovanović, B.(1989) Bull. Obs. Astron. Belgrade, 141, pp. 7.

Jenkins, L. F. (1952) Gen. Cat. of Trigonometric Stel. Parallaxes, Yale University Obs.

Melbourne, W., et al. (1983) Project Merit Standards, USNO Circular, No. 167.

Roeser, S., Bastian, U. (1991) Positions and Proper Motions (PPM) Star Catalogue, Astron. Rechen-Institut, Heidelberg.

Ševarlić, B., Teleki, G. (1960) Bull. Obs. Astron. Belgrade, 24, No. 3/4, pp. 19.

Vondrák, J., Feissel, M., Essaifi, N. (1992) Astron. Astrophys., 262, pp. 329.

Wilson, R. E. (1953) Gen. Cat. of Stel. Radial Velocities, Carnegie Institution of Washington Publ., Mount Wilson Obs., 601. 